\title{
Enhancement of UV Excited Photoluminescence by Fabry-Perot Microcavity
}

\author{
Chunxian Tao, ${ }^{1}$ Jun Ruan, ${ }^{1}$ Dong Liang, ${ }^{2}$ Zhaoxia Han, ${ }^{1}$ Liang He, ${ }^{1}$ Ruijin Hong, \\ Xiao Cui, ${ }^{1}$ and Dawei Zhang ${ }^{1}$ \\ ${ }^{1}$ Engineering Research Center of Optical Instrument and System, Ministry of Education and Shanghai Key Lab of \\ Modern Optical System, University of Shanghai for Science and Technology, No. 516 Jungong Road, Shanghai 200093, China \\ ${ }^{2}$ College of Communication and Art Design, University of Shanghai for Science and Technology, No. 516 Jungong Road, \\ Shanghai 200093, China
}

Correspondence should be addressed to Chunxian Tao; tao@usst.edu.cn

Received 22 July 2015; Revised 20 November 2015; Accepted 26 November 2015

Academic Editor: Khalique Ahmed

Copyright (C) 2015 Chunxian Tao et al. This is an open access article distributed under the Creative Commons Attribution License, which permits unrestricted use, distribution, and reproduction in any medium, provided the original work is properly cited.

\begin{abstract}
A light-emitting microcavity with the structure of dielectric mirror/phosphor coating/dielectric mirror for the enhancement of PL efficiency excited under UV light was designed and fabricated. The fluorescence emission of Lumogen S0795 coating within microcavity structure is significantly enhanced compared with the coating on bare substrate. The measurement results indicate the possibility of developing front illuminated CCD based on optical resonant cavity for UV-visible imaging with higher sensitivity.
\end{abstract}

\section{Introduction}

One-dimensional photonic crystal is a periodic structure that consisted with different refractive index of dielectric materials $[1,2]$. When the light comes through this medium, the light of certain frequency range will be suppressed and formed into photonic band gap. It is similar to the band structure of the electrons in semiconductor material. The theoretical model [3] and the application of photonic crystals are very extensive $[4,5]$, and the most common and easiest preparation of one-dimensional photonic crystal is usually prepared by physical vapor deposition method of multilayer structure with defect, which means a Fabry-Perot cavity sized for a light wave on the one-dimensional direction vertical cavity surface.

In recent years, because the significance on basic physical electrodynamics research, the research on emission properties of any kinds of luminous materials in microcavity has been paid more and more attentions [6-8]. Particularly the plane microcavity made of organic materials has been more conspicuous [9-11]. Lumogen, a kind of organic fluorescent material with UV-frequency conversion ability, can be used in CCD device to detect the ultraviolet spectrum area. In this paper, we adopted Lumogen as photonic crystal defect area. Based on the characteristics of stimulated emission of the Lumogen material, we design an artificial crystal structure in the form of Fabry-Perot microcavity to improve the photoluminescence efficiency of the fluorescent material, which makes CCD device have a greatly improvement in detective ability and sensitivity to ultraviolet signal.

\section{Simulation}

When we come up with the traditional multilayer high reflective film, there only exists light cut-off region in the center frequency which is represented by $\omega_{0}$ and its odd number of times, and there is no light cut-off region at the incident for $\omega_{0}$ even times, as shown in Figure 1. From the perspective of photonic crystals, there are band gap structures in general frequency $\omega_{0}$ integer times. Namely, when $k *$ $d=n * \pi$, there exists a band gap. If the light comes through a vertical incidence from medium A to medium B, the reflectivity can be given by Fresnel formula:

$$
\begin{aligned}
R & =r_{a b} r_{a b}^{*}, \\
r_{a b} & =\frac{n_{b}-n_{a}}{n_{b}+n_{a}},
\end{aligned}
$$




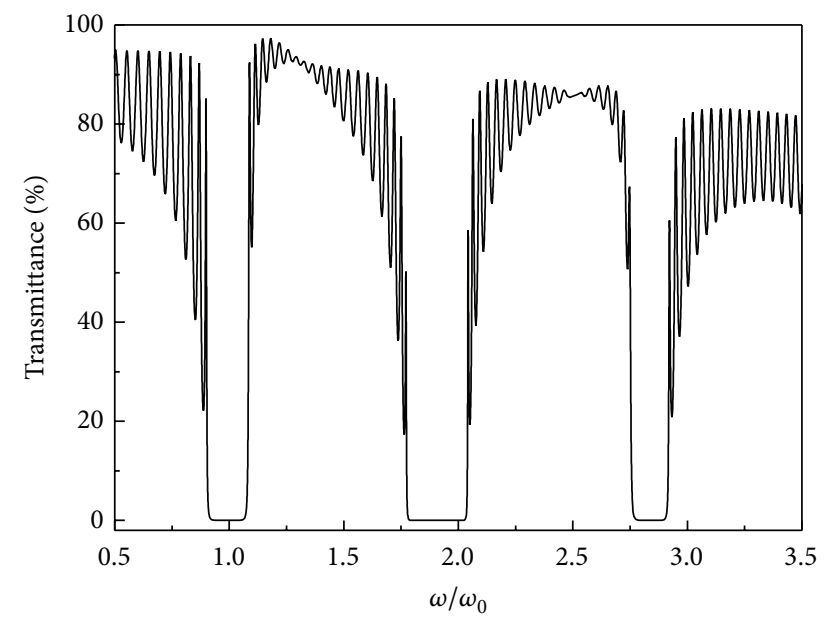

Figure 1: Transmission spectrum of one-dimensional photonic crystal at $0^{\circ}$ incidence.

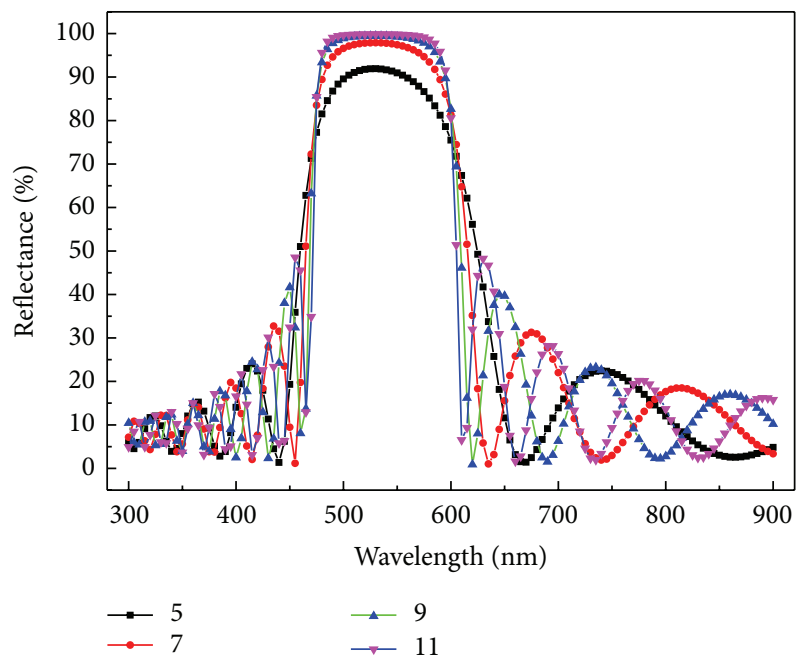

FIgURE 2: Reflective spectrum of $5,7,9$, and 11 periodic dielectric multilayer.

where $n_{a}$ and $n_{b}$ are the refractive indexes of two different media. For the limited periodic structure material, we could use the transfer matrix method to get the transfer characteristic of the membrane reactor. By the above theory the reflective spectra of the films with period of $5,7,9$, and 11, respectively, can be obtained, as shown in Figure 2. The period mentioned here refers to a pair of membrane consisting of two different mediums A and B. Theoretically the photonic crystal should have infinite structure, but in practice, the width of the energy gap basically no longer changes as long as there is a certain number of periods, as shown in Figure 2. So the traditional high antimembrane can be regarded as a kind of onedimensional photonic crystal, and the finite membrane stack should also be considered a special case of one-dimensional photonic crystal. So the research of fluorescent material enhancement can be discussed combined with thin solid film optical theory with the theory of the photonic crystal.

When the fluorescent material is doped in photonic crystal, an impurities defect mode in the photonic band gap

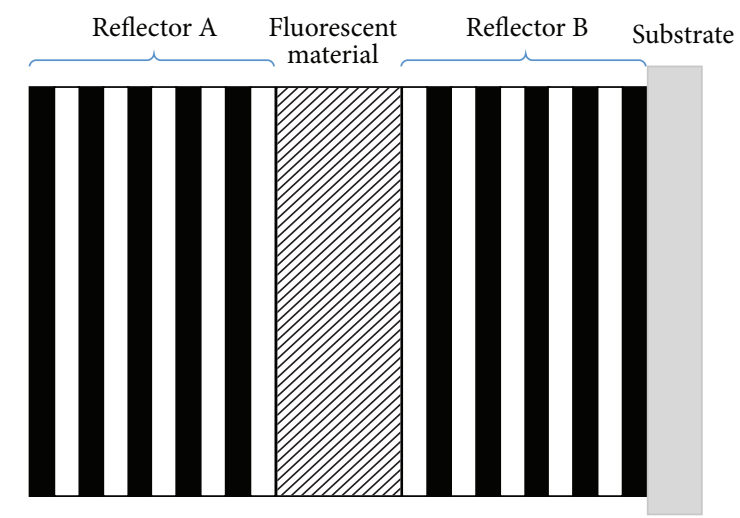

FIGURE 3: Structure design of one-dimensional photonic crystal.

would be created. It would change the light propagation mode and the density of states. The enhancement ratio can be obtained by comparing the density of states in free space and photonic crystal. According to the gold Fermi's rule, the ratio of spontaneous emission in photonic crystal and free space was decided by the ratio of the density of states. The density of states in free space was given by

$$
\rho_{i}(\omega)=\frac{1}{V} \cdot \frac{d N}{d V}=\frac{\omega^{2}}{\pi^{2} C^{3}}=\frac{4 n}{c},
$$

where $n$ was the refractive index of medium, $c$ was the speed of light in vacuum, and $V$ was the mode volume. $C$ is associated with parameters and frequency of the dipole of the incident photon. The density of states in photonic crystal was given by

$$
\rho_{c}(\omega)=\frac{1}{\delta \omega V}=\frac{n}{c} \cdot \sqrt{10 F}, \quad F=\frac{4 R}{(1-R)^{2}},
$$

where $F$ was the degree coefficient and $R$ was the reflectivity of the mirror. There is only single mode where bandwidth is $\delta \omega$ and the frequency is $\omega$. So the ratio of the state's density of photonic crystal and free space that was represented by $M$ was given by

$$
M=\frac{\sqrt{40 R}}{4(1-R)}
$$

So the value of $M$ was decided by the reflectance $R$ and is greater than 1 in the case of $R>90 \%$. That meant the photonic crystal can improve the rate of spontaneous emission and one dimensional photonic crystal structure can effectively enhance the luminous efficiency of organic light-emitting materials.

As shown in Figure 3, there was a fluorescent layer as defect in our designed photonic crystal structure. In order to make the UV detecting light incentive fluorescent material, the reflective mirror A was designed that the UV band light could pass through and a certain band gap with the center wavelength of $525 \mathrm{~nm}$. While the reflective mirror B was only designed with a band gap of certain bandwidth in the center of $525 \mathrm{~nm}$. So this was a photonic crystal with asymmetric 


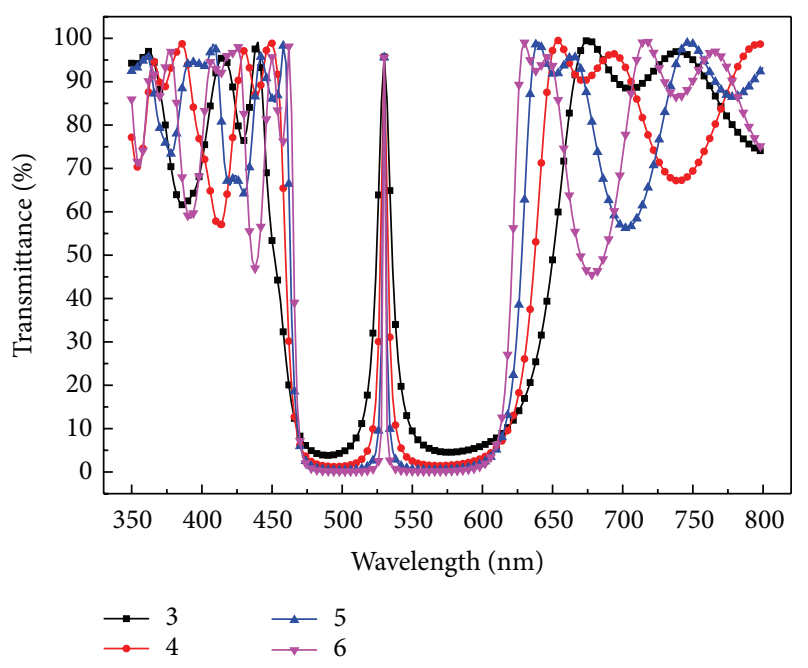

FIGURE 4: Transmitted spectrum of one-dimensional photonic crystal with different periods.

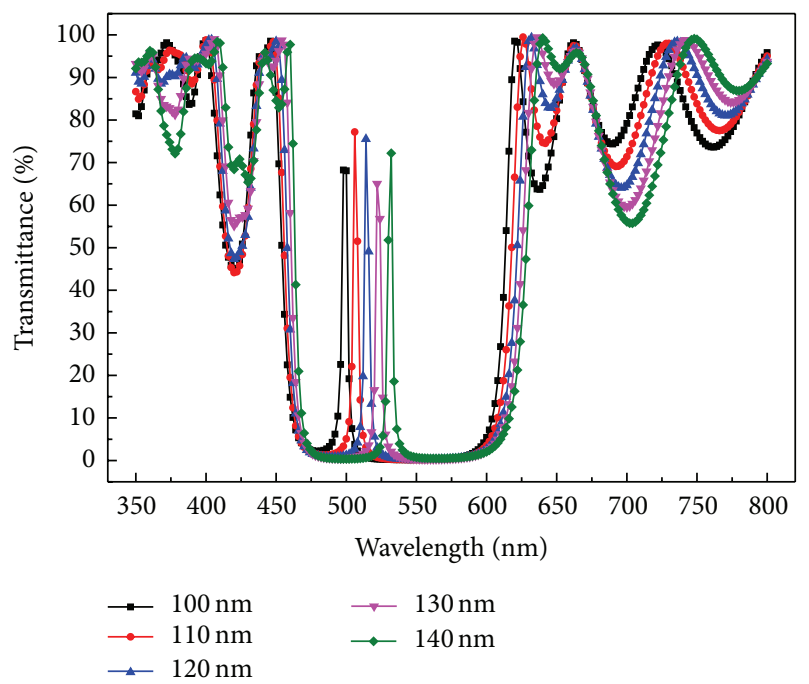

FIGURE 5: Transmitted spectrum of one-dimensional photonic crystal with different thickness of defect layer.

structure. According to the characteristics of photonic crystal, the transmission spectrum was very sensitive to the influence that geometric structure brought in, including the period and thickness of the defect.

As shown in Figure 4, a narrow transmission peak can be observed in the center of the photonic band gap. The photonic crystal structure with fewer periods had a shallower forbidden band gap and greater bandwidth. This meant that the impurity level was created in the photonic band gap and the transmission peak can be used to guide the visible emitting light of the organic material out.

The transmission spectra of the photonic crystal with different thickness of defect layer (100, 110, 120, 130, and $140 \mathrm{~nm}$ ) were shown in Figure 5. It could be found that there was little impact on the spectrum with different thickness compared with that of Figure 4 . Even the location of the transmission peak had drifted to the longer wavelength with

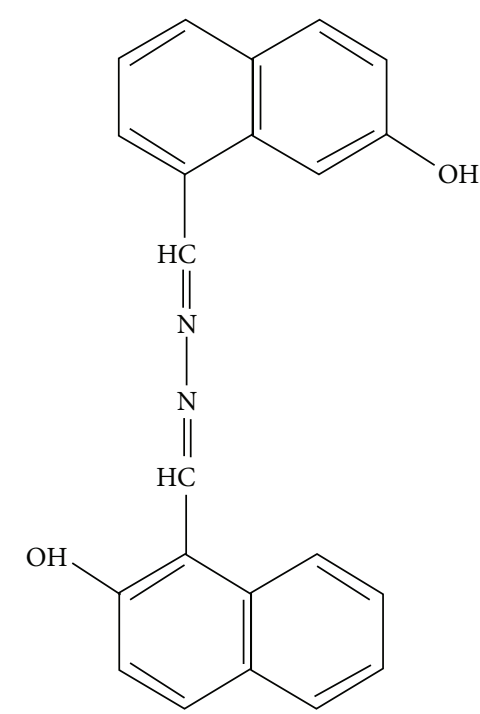

FIgURE 6: Chemical structure formula of Lumogen S0790.

the thickness increasing of the defect layers, the emission peaks of fluorescent material still fell into the response band the Si-based detectors. It is of great help for us to fabricate the UV-sensitive devices based on the photonic crystal with fluorescent material.

\section{Experiment}

The fluorescent material used for defect layer was Lumogen S0790 made by company BASF. The chemical structure formula was shown in Figure 6. There were fluorescent phenyl groups in the molecular and hydroxyl group that had function of fluorescent auxochrome. This structure could make the internal electronic of the molecular in transition, so the molecular group absorbed ultraviolet light and emitted visible light at the same time. It was found that this material emitted strong visible light near $525 \mathrm{~nm}$ under the irradiation of wide range of ultraviolet light.

Based on the properties of the organic fluorescent material, the photonic crystal was designed as follows: air | top reflective mirror | defect layer | bottom reflective mirror | substrate. The structure of the top reflective mirror was $\left(\mathrm{H}^{\prime} \mathrm{L}^{\prime}\right)^{\wedge m} \mathrm{H}^{\prime}$, and the bottom reflective mirror was $(\mathrm{HL})^{\wedge n} \mathrm{H}$. H was one layer of high refractive index material, $\mathrm{ZrO}_{2}$, while $\mathrm{L}$ was one low, $\mathrm{SiO}_{2} . \mathrm{H}^{\prime}$ and $\mathrm{L}^{\prime}$ were not a quarter attribute film system and $m$ and $n$ were the number of the repeat period.

The reflective mirrors were prepared by electronic beam evaporation method, with baking temperature $200^{\circ} \mathrm{C}$ and ultimate pressure $1 * 10^{-4} \mathrm{~Pa}$. And the defect layer was prepared by resistance heat evaporation, with baking temperature $40-60^{\circ} \mathrm{C}$ and ultimate pressure $8 * 10^{-4} \mathrm{~Pa}$.

\section{Discussion}

The transmittance spectra of the mirror sample were obtained by the spectrophotometer (PerkinElmer, Lambda 1050). The transmission spectrum of the top reflective mirror was shown 


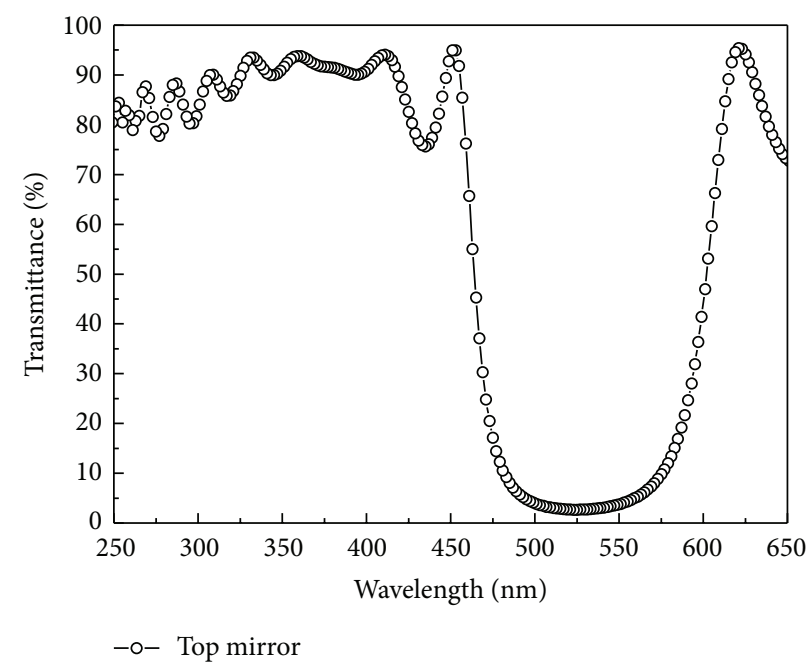

FIGURE 7: The transmitted spectrum of top HR mirror.

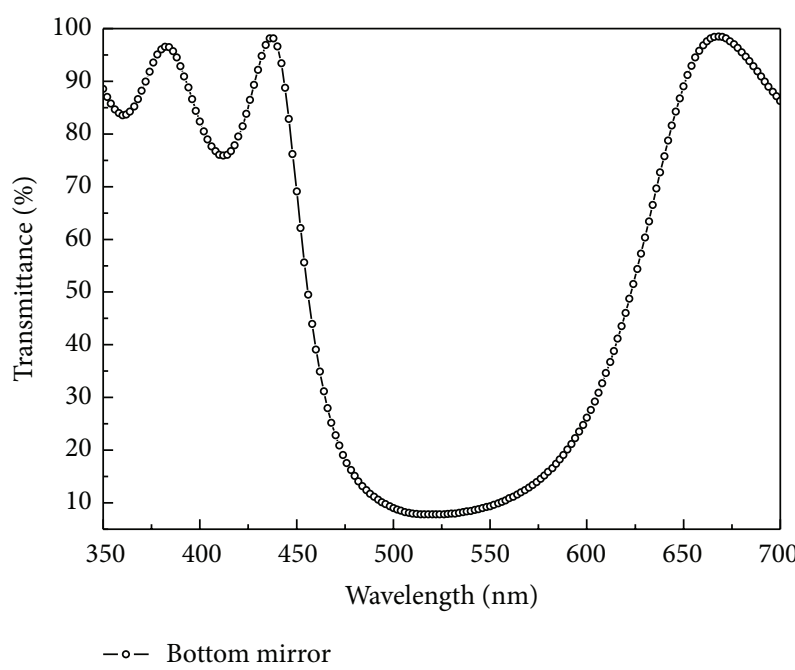

FIgURE 8: The transmitted spectrum of bottom HR mirror.

in Figure 7. The average transmittance at $250-400 \mathrm{~nm}$ was about $85 \%$, which satisfied the input of the ultraviolet light to the microcavity. The bandwidth was nearly $70 \mathrm{~nm}$ and the average transmittance was $4 \%$. The transmission spectrum of the bottom reflective mirror was shown in Figure 8. The bandwidth was nearly $75 \mathrm{~nm}$ and the average transmittance was $7 \%$.

The transmission spectrum of the photonic crystal in the form of Fabry-Perot microcavity was shown in Figure 9. The transmittance of the peak was nearly $60.1 \%$ and the full width half maximum was nearly $11 \mathrm{~nm}$, while width of the two cut-off bands was $80 \mathrm{~nm}$ and $54 \mathrm{~nm}$, respectively. And the transmittance of the cut-off bands was only $2 \%$ and $1 \%$, respectively.

The photoluminescence (PL) spectra of the photonic crystal were shown in Figure 10, which were obtained by fluorescence spectrometer (Horiba, Aqualog DualFL-UV-NIR). The studied excitation wavelength was $250 \mathrm{~nm}, 300 \mathrm{~nm}$, and

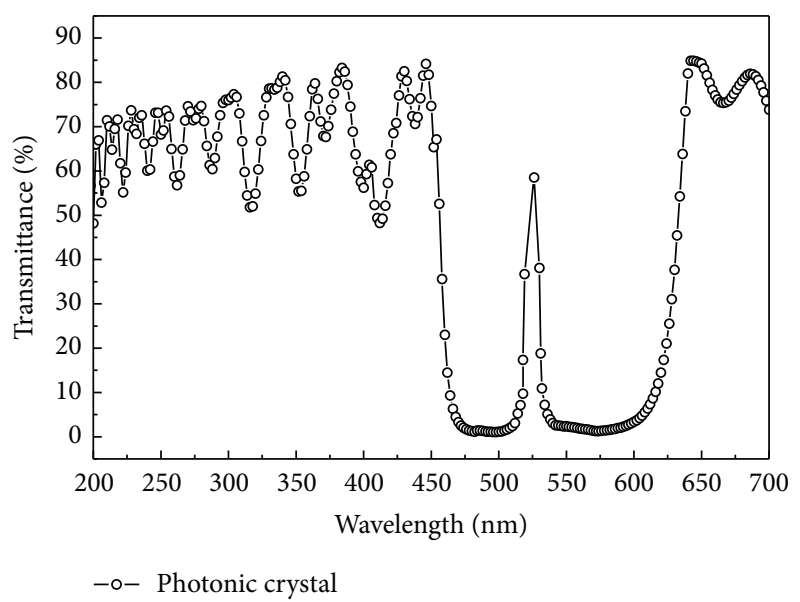

FIGURE 9: The transmitted spectrum of one-dimensional photonic crystal.

$330 \mathrm{~nm}$, respectively. It was found that he PL efficiency was all enhanced effectively and obviously at the three selected ultraviolet wavelength. The peak intensity increased nearly by $62 \%$ at the excitation of $250 \mathrm{~nm}, 90 \%$ at $300 \mathrm{~nm}$, and $57 \%$ at $330 \mathrm{~nm}$. By integration of the intensity of the emission spectrum, there was a greater enhancement of PL efficiency and conversion efficiency for UV to visible light.

\section{Conclusion}

In this paper, a one-dimensional photonic crystal in the form of Fabry-Perot microcavity with the structure of dielectric mirror and phosphor coating was simulated and fabricated. The photoluminescence efficiency of the fluorescent material was investigated. By emission spectrum, we could find that the photoluminescence intensity of Lumogen fluorescent material has been significantly improved. Our study provides a feasibility of developing front illuminated CCD devices for UV light detection with higher sensitivity. 


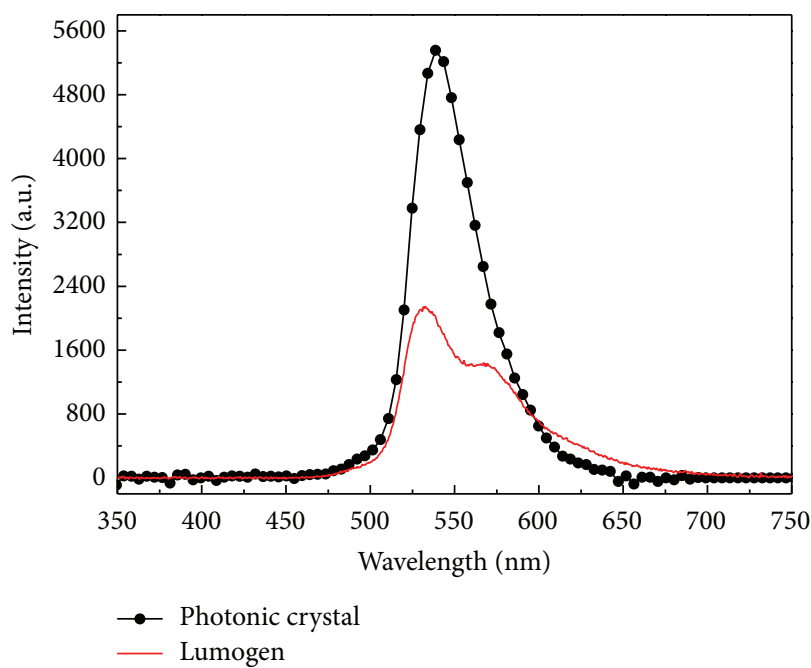

(a)

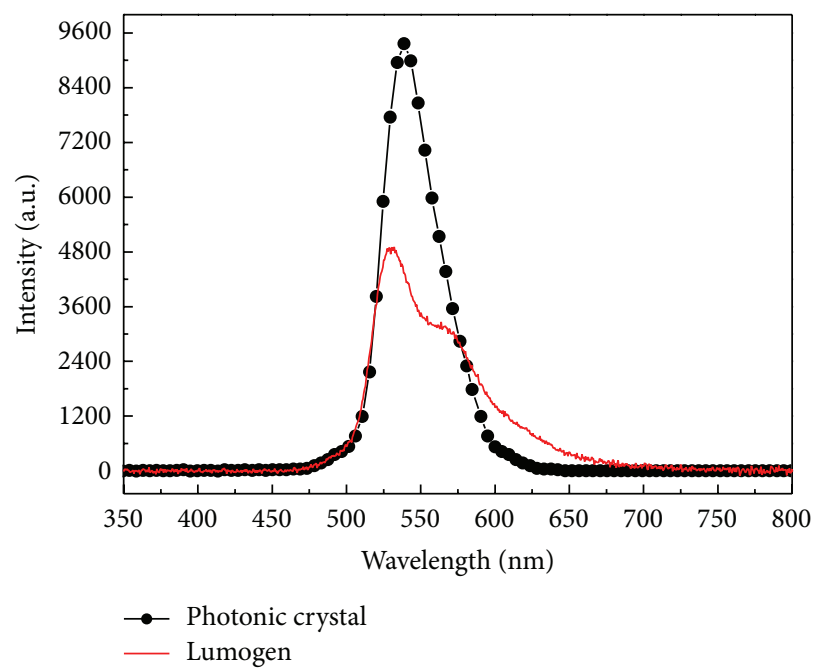

(b)

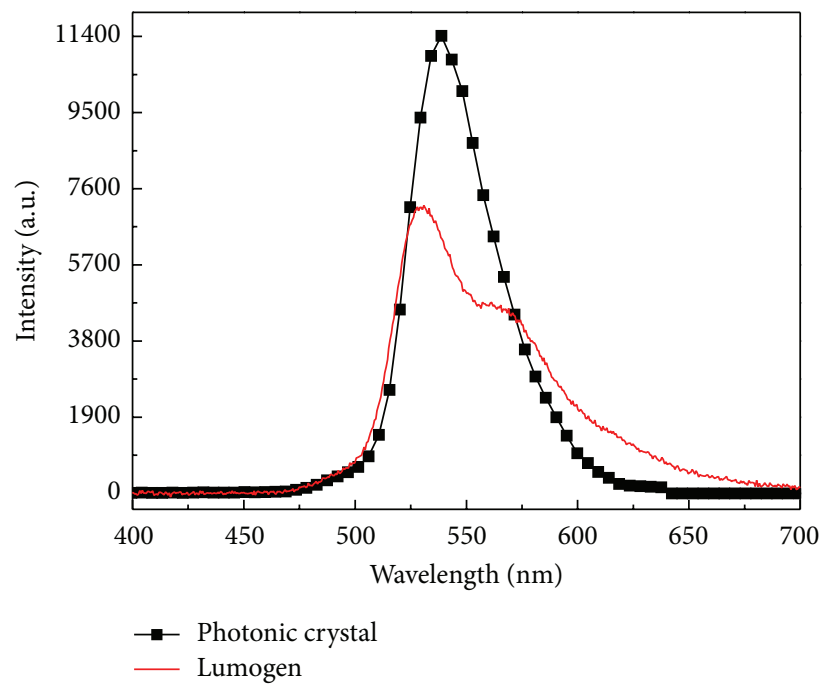

(c)

Figure 10: PL spectrum of one-dimensional photonic crystal excited at 250, 300, $330 \mathrm{~nm}$.

\section{Conflict of Interests}

The authors declare that there is no conflict of interests regarding the publication of this paper.

\section{Acknowledgments}

This work was partially supported by the National Science Instrument Important Project (2011YQ14014704), Shanghai Municipal Science Instrument Important Project (14142200902), and National Natural Science Foundation of China (61378060 and 61205156).

\section{References}

[1] E. Yablonoviteh, "Inhibited spontaneous emission in solid-state physics and electronics," Physical Review Letters, vol. 58, no. 20, pp. 2059-2061, 1987.
[2] S. John, "Strong localization of photons in certain disordered dielectric superlattices," Physical Review Letters, vol. 58, no. 23, pp. 2486-2489, 1987.

[3] M. M. Voronov, E. L. Ivchenko, M. V. Erementchouk, L. I. Deych, and A. A. Lisyansky, "Photoluminescence spectroscopy of one-dimensional resonant photonic crystals," Journal of Luminescence, vol. 125, no. 1-2, pp. 112-117, 2007.

[4] I. D. Block, P. C. Mathias, S. I. Jones, L. O. Vodkin, and B. T. Cunningham, "Optimizing the spatial resolution of photonic crystal label-free imaging," Applied Optics, vol. 48, no. 34, pp. 6567-6574, 2009.

[5] V. Chaudhery, M. Lu, C.-S. Huang et al., "Line-scanning detection instrument for photonic crystal enhanced fluorescence," Optics Letters, vol. 37, no. 13, pp. 2565-2567, 2012.

[6] N. Takada, T. Tsutsui, and S. Saito, "Control of emission characteristics in organic thin-film electroluminescent diodes using an optical-microcavity structure," Applied Physics Letters, vol. 63, no. 15, pp. 2032-2034, 1993. 
[7] P. Schouwink, H. von Berlepsch, L. Dähne, and R. F. Mahrt, "Observation of strong exciton-photon coupling in an organic microcavity in transmission and photoluminescence," Journal of Luminescence, vol. 94-95, pp. 821-826, 2001.

[8] X. Y. Liu, L. X. Wang, Y. Liu et al., "Spontaneous emission properties of organic film in plane optical microcavity," Thin Solid Films, vol. 363, no. 1, pp. 204-207, 2000.

[9] B. Masenelli, S. Callard, A. Gagnaire, and J. Joseph, "Fabrication and characterization of organic semiconductor-based microcavities," Thin Solid Films, vol. 364, no. 1, pp. 264-268, 2000.

[10] S. Dirr, S. Wiese, H.-H. Johannes et al., "Luminescence enhancement in microcavity organic multilayer structures," Synthetic Metals, vol. 91, no. 1-3, pp. 53-56, 1997.

[11] G. O. Mueller, R. Mueller-Mach, E. Alinsog, H. Lee, and D. Harrison, "Microcavity effects in thin-film electroluminescence," Journal of Luminescence, vol. 72-74, pp. 1002-1004, 1997. 

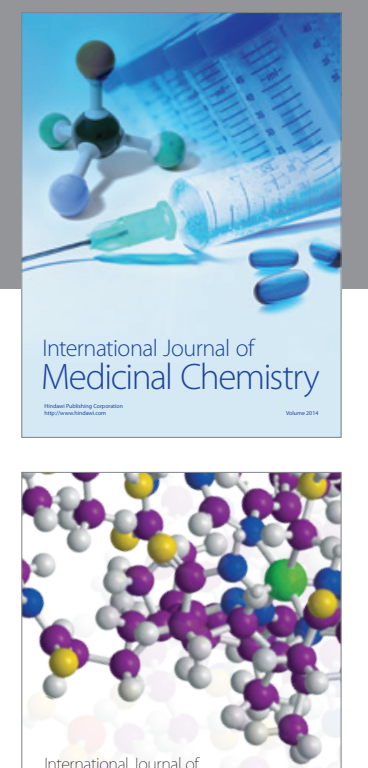

\section{Carbohydrate} Chemistry

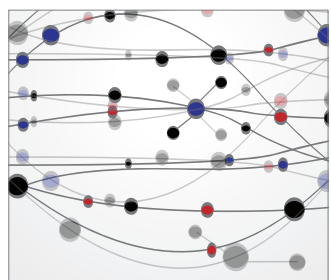

The Scientific World Journal
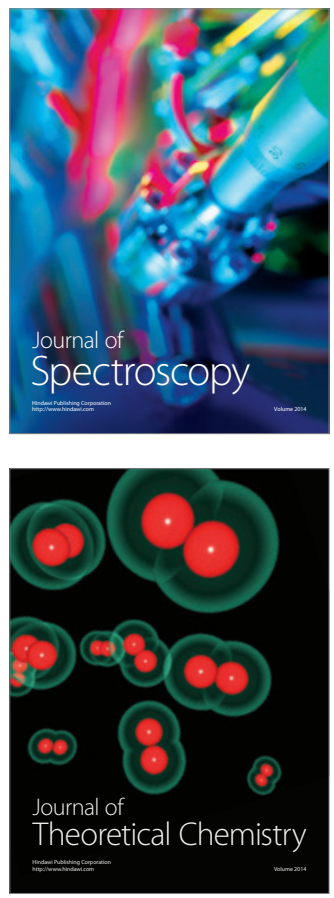
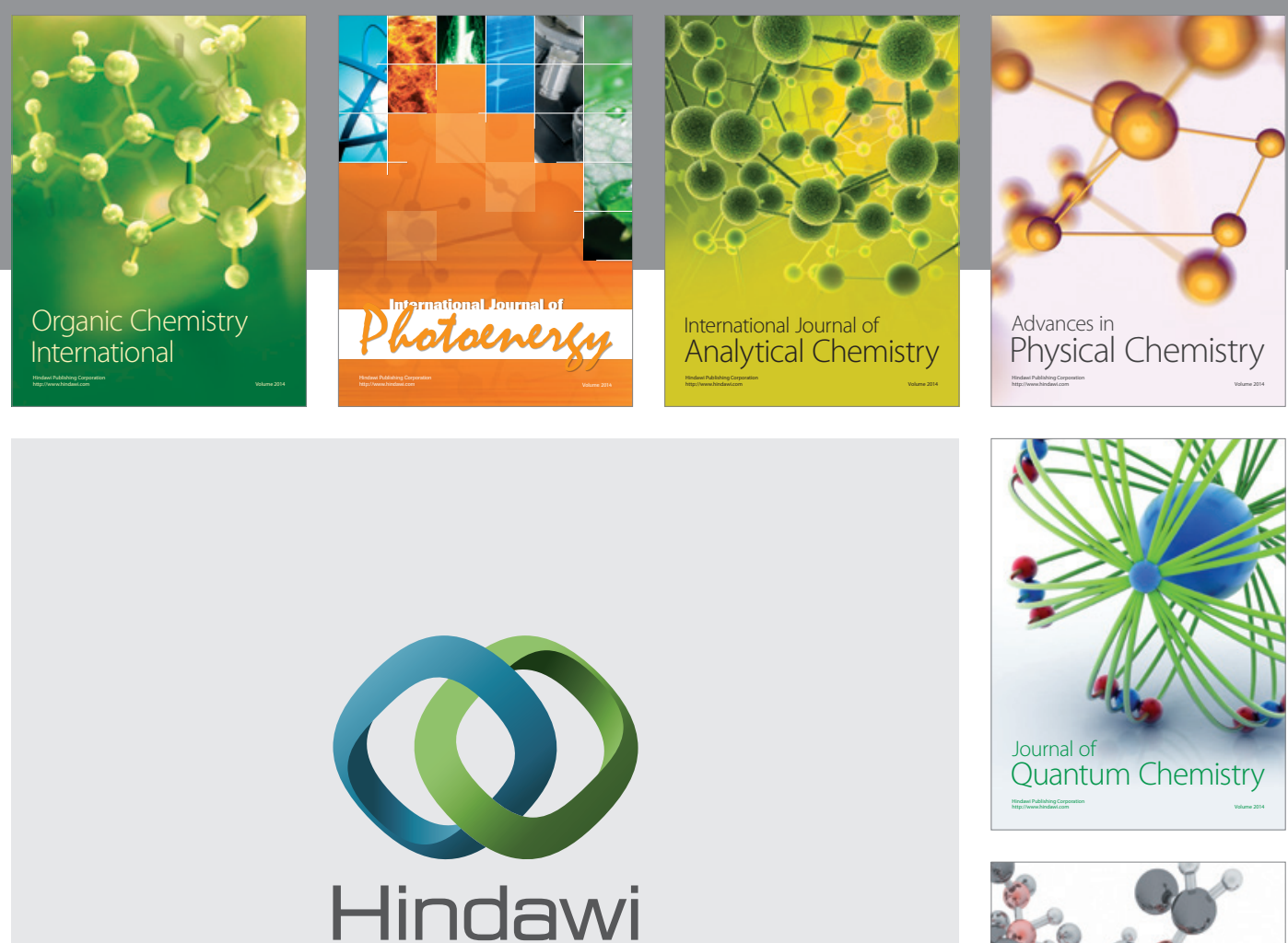

Submit your manuscripts at

http://www.hindawi.com

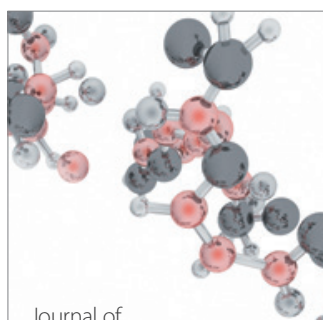

Analytical Methods

in Chemistry

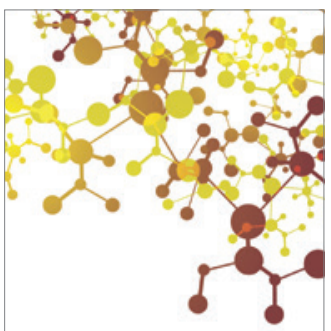

Journal of

Applied Chemistry

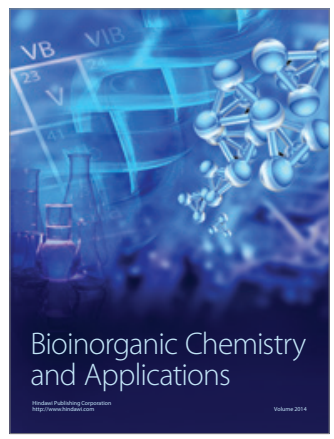

Inorganic Chemistry
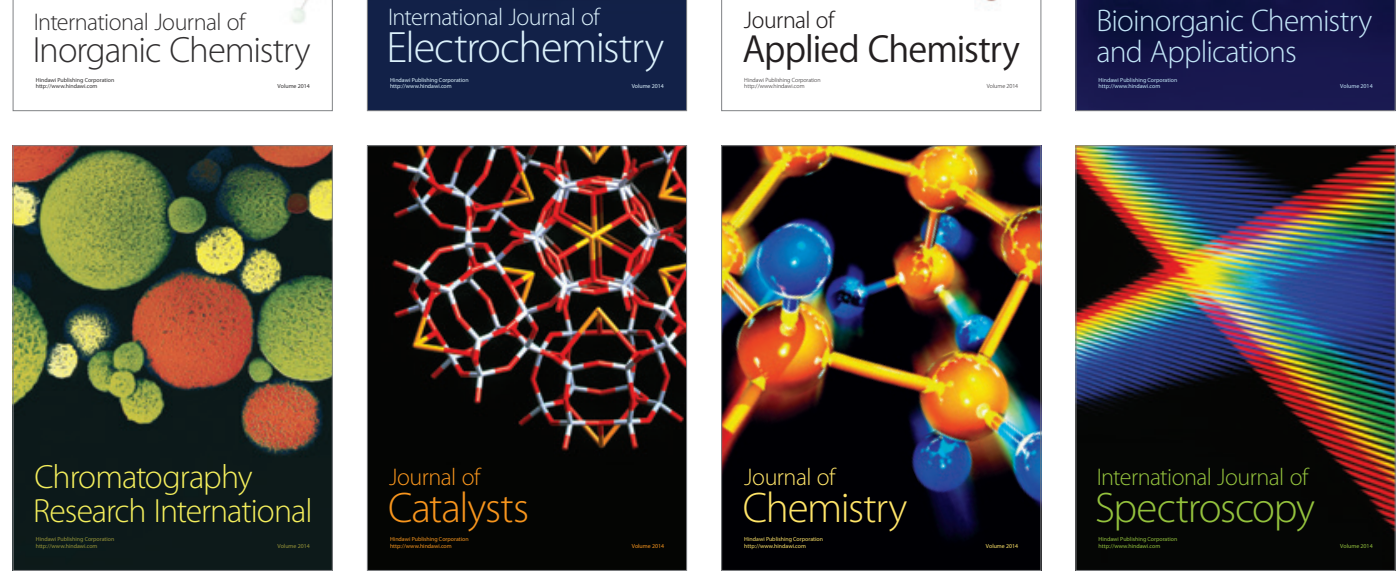